\title{
Ontologias como suporte à avaliação enquanto tarefa intensiva em conhecimento: uma revisão bibliométrica
}

Vanderlei Freitas Junior

\begin{abstract}
Doutorando em Engenharia e Gestão do Conhecimento pela Universidade Federal de Santa Catarina, com foco para a linha de Engenharia do Conhecimento. Mestre em Educação pela Universidade Federal Rural do Rio de Janeiro (UFRRJ). Graduado em Ciência da Computação pela Universidade do Sul de Santa Catarina (UNISUL). Professor do Instituto Federal de Educação, Ciência e Tecnologia Catarinense, Campus Sombrio - SC.
\end{abstract}

Alexandre Leopoldo Gonçalves

Doutorado e mestrado em Engenharia de Produção pela Universidade Federal de Santa Catarina em 2000 e 2006. Graduado em Ciência da Computação pela Fundação Universidade Regional de Blumenau (1997). Professor Adjunto da Universidade Federal de Santa Catarina e Professor Permanente do Programa de PósGraduação em Engenharia e Gestão do Conhecimento da UFSC. Bolsista CNPq Brasil.

http://dx.doi.org/10.1590/1981-5344/1930

Objetiva analisar as publicações internacionais acerca do emprego das ontologias como suporte à tarefa avaliação enquanto tarefa intensiva em conhecimento. Para isso, foi realizada uma pesquisa bibliométrica em três bases de dados internacionais. Essa pesquisa foi desenvolvida em duas etapas: (i) busca sistemática da literatura, e (ii) análise e síntese das informações bibliométricas. Foram localizados 38 trabalhos, escritos por 106 autores, oriundos de 44 instituições, de 18 países diferentes. A partir da identificação destes estudos o presente trabalho contribui para a compreensão das possibilidades de aplicação das ontologias para o suporte à tarefa de avaliação enquanto tarefa intensiva em conhecimento, realizando o mapeamento das publicações internacionais desta temática e evidenciando a pequena quantidade de estudos que as relacionam. 
Palavras-chave: Ontologia; Avaliação; Tarefa intensiva em conhecimento.

\title{
Ontologies in support of evaluation as knowledge-intensive task: a bibliometric review
}

\begin{abstract}
This paper aims to analyze the international publications about the use of ontologies as a support to evaluation task as a knowledge-intensive task. For this, we performed a bibliometric survey in three international databases. This research was conducted in two stages: (i) systematic literature search, and (ii) analysis and synthesis of bibliometric information. We located 38 papers, written by 106 authors, coming from 44 institutions in 18 different countries. From the identification of these studies, the present work contributes to understanding of the possibilities of application of ontologies for supporting the evaluation task as knowledge-intensive task, performing the mapping of international publications of this theme and highlighting the small number of studies that relate it.
\end{abstract}

Key-words: Ontology; Assessment; Knowledge-Intensive Task.

Recebido em 26.11.2013Aceito em 10.04.2014

\section{Introdução}

Pesquisadores têm apontado para o relevante papel das ontologias na representação do conhecimento de um determinado domínio e no desenvolvimento de sistemas baseados em conhecimento. Ugwu, Anumba e Thorpe (2005) afirmam que "as ontologias são as fundações nas quais os resolvedores de problemas intensivos em conhecimento se apoiam". Inclusive, diante do grande volume de fonte de informações disponíveis e a consequente dificuldade de recuperação destas informações, pesquisadores da área de Ciência da Informação tem visto nas ontologias uma importante ferramenta para a organização e recuperação da informação (ALMEIDA, BAX, 2003; RAUTENBERG, 2010).

Por outro lado, a metodologia CommonKADS, considerada uma das mais maduras para o desenvolvimento de sistemas baseados em conhecimento (GOBIN, 2011), preocupa-se com a necessidade de reutilização de seus componentes, tornando este processo de 
desenvolvimento menos demorado. Neste sentido, Schreiber et al (2002) estabelece um conjunto de modelos de tarefas (task templates), que são modelos de conhecimento parciais nos quais as inferências e o conhecimento da tarefa são especificados. Nestes modelos, as principais tarefas intensivas em conhecimento são descritas, permitindo que sejam reutilizadas pelos engenheiros do conhecimento como um padrão para novas aplicações.

Dentre as tarefas intensivas em conhecimento descritas por Schreiber (2002), encontra-se a tarefa de avaliação. Esta tarefa tem por objetivo encontrar uma categoria de decisão para um caso baseada em um conjunto de normas específicas do domínio.

Neste sentido, o objetivo do presente estudo é o de investigar o uso de ontologias como suporte à tarefa intensiva em conhecimento de avaliação, realizando um mapeamento das publicações científicas nesta temática.

Este artigo está estruturado como segue: a seção 2 apresenta os aspectos conceituais deste estudo, seguida da seção 3 , que delineia os aspectos metodológicos da pesquisa. A seção 4 apresenta os resultados da pesquisa bibliométrica, enquanto a seção 5 realiza o delineamento dos estudos recuperados a partir da busca realizada. A seção 6 apresenta as considerações finais, precedendo as referências, que são apontadas na seção 7.

\section{Aspectos conceituais}

A engenharia do conhecimento, desde os seus primórdios, enfoca na utilização de metodologias e técnicas formais que permitam desenvolver sistemas baseados em conhecimento, de forma sistêmica e controlada (STUDER et al, 2000). É uma disciplina derivada da Inteligência Artificial, com o objetivo de construir sistemas de conhecimento, amparando-se em metodologias, técnicas, linguagens e ferramentas para extração, codificação, representação e uso de conhecimento (RAUTENBERG et al, 2008; SCHREIBER et al, 2002), com especial destaque para as tarefas intensivas em conhecimento.

As tarefas intensivas em conhecimento podem ser definidas como tarefas que necessitam de alto nível de conhecimento especializado para a sua execução. São, em geral, executadas por especialistas, importantes quando do processo de automação das tarefas intensivas em conhecimento em razão de sua ampla experiência na execução deste tipo de tarefa (KOENDERINK, 2010).

Para Koenderink (2010), uma tarefa intensiva em conhecimento pode ser decomposta em subtarefas, que por sua vez são representadas por uma sequência de componentes que claramente definem parte de uma tarefa. Cada um destes componentes são especificados a partir de três aspectos: (i) os conceitos de entrada, (ii) os conceitos de saída; e, (iii) uma descrição em alto nível do serviço.

Schreiber et al (2002) abordam o conceito de tarefas intensivas em conhecimento a partir da ideia de modelos de tarefas. Um 
modelo de tarefa, segundo os autores, fornece ao engenheiro do conhecimento as inferências e as tarefas que são tipicamente utilizadas para a solução de um tipo específico de problema.

As atividades intensivas em conhecimento, são classificadas por Schreiber et al (2002) como analíticas e sintéticas. Nas tarefas analíticas, verifica-se a entrada de dados de um sistema existente, e produz alguma caracterização deste sistema como saída. No caso das tarefas sintéticas, o sistema ainda é inexistente e seu propósito é construir uma descrição deste sistema. A Figura 1 apresenta a hierarquia dos tipos de tarefas intensivas em conhecimento descrita por Schreiber et al (2002) com base no tipo de problema a ser resolvido.

Figura 1 - Hierarquia das tarefas intensivas em conhecimento

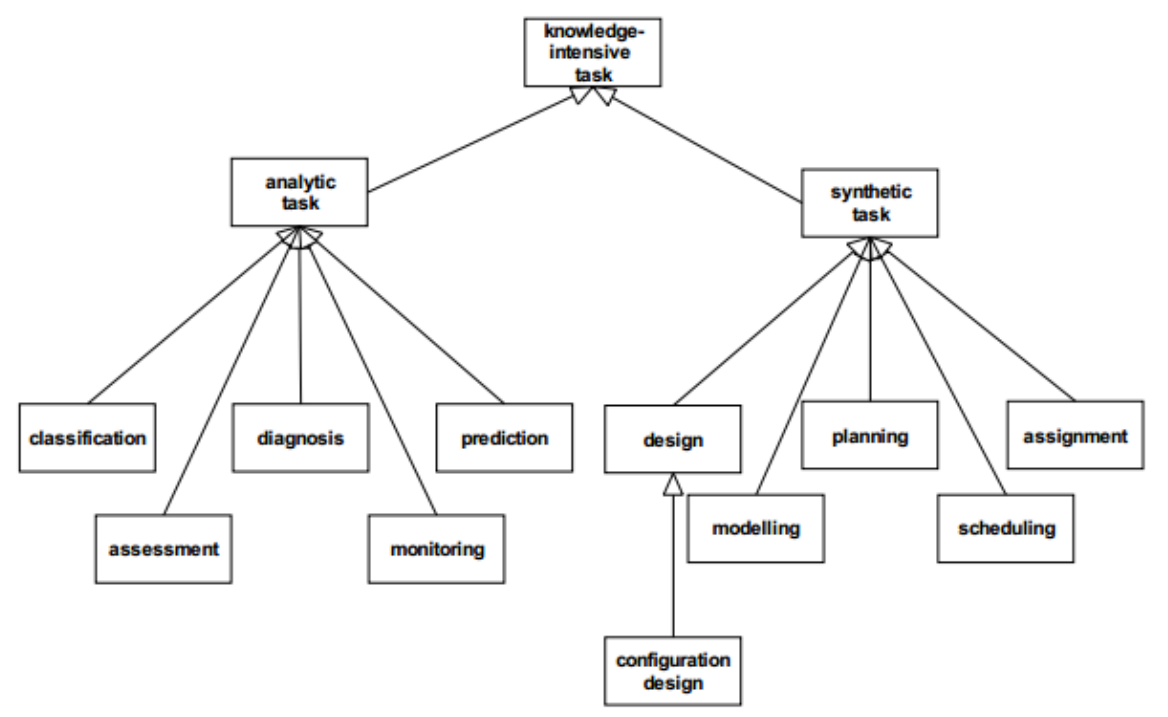

Fonte: Schreiber et al (2002)

Os autores apresentam exemplos de tarefas intensivas em conhecimento do tipo analítica e sintéticas. São tarefas analíticas: classificação, avaliação, diagnóstico, monitoramento, previsão. São tarefas sintéticas: projeto, modelagem, planejamento, programação, atribuição (SCHREIBER et al, 2002).

Nosso foco principal neste trabalho está na tarefa intensiva em conhecimento de avaliação. A avaliação, enquanto tarefa intensiva em conhecimento do tipo analítica tem como objetivo a localização de uma categoria de decisão para um caso baseado em um conjunto de normas específicas de um domínio (SCHREIBER et al, 2002).

A tarefa de avaliação (assessment) tem como entrada a descrição de um caso. Como saída, apresenta uma classe de decisão. 0 conhecimento envolvido no processo de avaliação é consubstanciado de critérios e normas. A avaliação tem como característica o fato de que é executada em um ponto específico do tempo, ao contrário da tarefa de monitoramento, por exemplo, que leva em consideração o fato de que o sistema muda ao longo do tempo, sendo executada repetidamente (SCHREIBER et al, 2002). 
Os autores exemplificam a tarefa de avaliação trazendo o caso da avaliação de empréstimos. Como entrada, tem-se o caso de uma pessoa solicitando o empréstimo, e a saída é a classe de decisão do tipo "sim/não/necessário maiores informações", indicando se o empréstimo deve ou não ser fornecido. O conhecimento envolvido no processo decisório desta tarefa é um conjunto de normas e critérios usados na avaliação de concessão de empréstimos.

Assim, tem-se que a tarefa de avaliação, de maneira genérica, é uma tarefa intensiva em conhecimento consubstanciada na análise dos dados de entrada a partir de um conhecimento prévio (normas ou critérios), culminando com a decisão fruto desta avaliação (sim/não). Como tarefa intensiva em conhecimento, pode ser aplicada nos mais diversos domínios.

A Engenharia do Conhecimento, por sua vez, estuda metodologias para a representação deste conhecimento, tendo apontado as ontologias como uma das principais formas de representar computacionalmente o conhecimento de um determinado domínio.

Ontologia é um termo criado em filosofia para designar o estudo de tudo aquilo que existe, bem como suas relações, mas que desde a década de 1990 vem sendo referenciado em áreas como Ciência da Computação e Ciência da Informação (ALMEIDA; MENDONÇA; AGANETTE, 2013). Para a primeira área, podem ser definidas como especificações explícitas dos tipos de recursos que existem e os relacionamentos possíveis entre eles, e instâncias específicas de conceitos na ontologia (ABRAMOVICH, 2005).

Uma ontologia também pode ser definida como sendo um conjunto de termos ordenados hierarquicamente para representar um domínio específico. Ela pode ser usada como um esqueleto para uma base de conhecimento onde são executados processos de inferência (raciocínio). O uso de uma ontologia permite então, a definição de um domínio no qual será possível trabalhar em determinada área específica, possibilitando a melhora no processo de extração de informação e o intercâmbio do conhecimento (GÓMEZ-PÉREZ, 1999).

Uma definição amplamente aceita por engenheiros de ontologias (GOBIN, 2011) é de autoria de Studer, Benjamins e Fensel (1998), que estabelece que a ontologia é "uma especificação formal e explícita de uma conceitualização compartilhada", tendo sido ampliada por Borst (1997) ao afirmar que as ontologias são "especificações formais de conceitualizações compartilhadas", vindo a culminar com sua versão mais aceita que seria "especificação formal e explícita de uma conceitualização compartilhada". Para Gobin (2011), esta definição baseia-se na ideia da conceitualização, isto é, uma versão simplificada do mundo real que quer ser representada, fornecendo uma visão comum e compartilhada de um domínio, que pode ser comunicada entre pessoas e sistemas.

Campos (2010) contribui afirmando que uma ontologia também pode ser empregada para a definição de um vocabulário comum para uma comunidade que precisa compartilhar informação, incluindo definições de conceitos deste domínio bem como suas relações, de forma 
a ser interpretados por máquina. Estas definições possibilitam a descrição de conteúdo semântico, permitindo que agentes inteligentes façam inferências a respeito dos conceitos explicitados na ontologia.

Para Dillon e Simmons (2008), as ontologias compartilham uma compreensão comum acerca da estrutura da informação entre pessoas ou agentes de software, mas não apenas isto, elas também possibilitam a reutilização do conhecimento de determinado domínio, explicitam as suposições deste domínio, separam o conhecimento de domínio do conhecimento operacional, além de permitirem a análise do conhecimento de domínio. A engenharia de ontologias, por sua vez, é definida pelos mesmos autores como um processo altamente colaborativo, posto que uma ontologia desenvolvida com toda precisão pouco serve se não for aceita pelos especialistas de domínio, que devem participar diretamente de seu desenvolvimento.

Assim, admitindo-se as ontologias como ferramentas para a representação computacional do conhecimento específico de um domínio, o presente estudo busca investigar a maneira como os estudos científicos apontam o uso das ontologias no suporte à tarefa de avaliação, enquanto tarefa intensiva em conhecimento.

\section{Procedimentos metodológicos}

O procedimento metodológico adotado para o desenvolvimento deste trabalho foi o método de pesquisa bibliométrica, o qual emprega técnicas e ferramentas baseadas em métodos estatísticos e matemáticos para mapear informações a partir de registros bibliográficos de documentos armazenados em bases de dados (SANTOS; KOBASCHI, 2009). Os procedimentos metodológicos estão descritos a seguir:

\subsection{Etapa 1: busca sistemática de literatura}

Para realizar a busca sistemática da literatura, a respeito do emprego de ontologias para suporte à tarefa de avaliação enquanto tarefa intensiva em conhecimento, foram selecionadas as seguintes palavraschave: ontologia (ontology), avaliação (assessment) e tarefa intensiva em conhecimento (knowledge-intensive task).

As expressões de busca utilizadas foram: "ontolog* AND assessment AND knowledge-intensive task*" e "ontolog* AND knowledge-intensive task*", esta última exclusivamente para a base IEEE.

A adequação da expressão de busca em razão das especificidades da base teve o objetivo de incluir o maior número possível de estudos relevantes que abordem a temática de interesse deste trabalho. Por outro lado, a adoção dos caracteres especiais "?" e "*" são fundamentais para este mesmo propósito, visto que permitem variações das expressões de pesquisa, abarcando grafias diferentes e plurais.

As buscas foram realizadas no período de dezembro de 2012 a janeiro de 2013, nas bases Web of Science, Scopus e IEEE, bases internacionais, multidisciplinares e de importância reconhecida 
cientificamente (ALMEIDA, 2006). A base IEEE foi também incluída em razão da grande quantidade de estudos indexados na área de Engenharia do Conhecimento, foco de interesse deste trabalho.

Optou-se, como princípio básico para a busca em todas as bases de dados, a consulta nos campos título, abstract e palavras-chaves e para aquelas que não permitiam esta escolha, realizou-se a busca pelo maior número de campos disponível. Não houve restrição temporal na busca realizada, recuperando registros do período compreendido entre o ano de 1999 e o ano de 2012.

As buscas iniciais nas bases selecionadas retornou 41 registros, conforme demonstrado na Tabela 1.

Tabela 1 - Estudos localizados por base

\begin{tabular}{lc}
\multicolumn{1}{c}{ BASES } & ESTUDOS LOCALIZADOS \\
\hline Web of Science & 3 \\
Scopus & 4 \\
IEEE & 34 \\
\hline \multicolumn{2}{r}{ TOTAL } \\
\hline Fonte: Os autores.
\end{tabular}

Em seguida todos os registros, recuperados a partir das primeiras buscas, foram exportados para o software EndNote, no qual foram realizados os procedimentos bibliométricos. Posteriormente, realizou-se a identificação dos estudos repetidos e exclusão dos estudos indexados sem autoria. A retirada destes estudos culminou com a seleção de 38 trabalhos, distribuídos conforme demonstra a Tabela 2.

Tabela 2 - Estudos selecionados por base

\begin{tabular}{lc}
\multicolumn{1}{c}{ BASES } & ESTUDOS LOCALIZADOS \\
\hline Web of Science & 2 \\
Scopus & 2 \\
IEEE & 34 \\
\hline \multicolumn{2}{r}{ TOTAL } \\
\hline Fonte: Os autores.
\end{tabular}

Estes 38 trabalhos foram adotados, então, como sendo o conjunto principal de publicações, o qual foi utilizado para todas as análises bibliométricas posteriores.

\subsection{Etapa 2: análise e síntese das informações bibliométricas}

A partir da padronização dos dados no software EndNote, foi possível proceder a análise bibliométrica dos estudos recuperados, por meio de consultas e contagens de frequência.

Os 38 trabalhos identificados no levantamento bibliométrico foram classificados e analisados considerando as relações com a temática de ontologias e avaliação enquanto tarefa intensiva em conhecimento. Foram utilizados os seguintes indicadores bibliométricos, para realizar as análises 
e sínteses dos trabalho: distribuição temporal dos 38 trabalhos selecionados (publicações por ano); principais autores, instituições e países; principais palavras-chave; e os trabalhos mais citados.

\section{Resultados}

Os trabalhos analisados são compostos por um total de 38 estudos, obtidos a partir de 03 fontes (base de dados de publicações científicas) e após a aplicação de todos os critérios definidos previamente para exclusão. Estes trabalhos foram escritos por 106 autores, vinculados a 44 instituições diferentes, provenientes de 18 países. E, foram utilizadas 711 palavras-chave para identificar e indexar as publicações. A Tabela 3 apresenta os dados bibliométricos gerais acerca das publicações analisadas.

Tabela 3 - Dados bibliométricos gerais

\begin{tabular}{lc}
\hline \multicolumn{1}{c}{ Dados Bibliométricos } & Frequência \\
\hline Publicações & 38 \\
Repetidos & 3 \\
Fontes de publicação* & 3 \\
Autores & 106 \\
Instituições & 44 \\
Países & 18 \\
Palavras-chave & 711 \\
\hline
\end{tabular}

Fonte: Os autores.

A partir do levantamento geral passa-se à descrição dos demais resultados bibliométricos, detalhando-os de acordo com os elementos demonstrados na Tabela 3.

\subsection{Distribuição temporal dos trabalhos selecionados (publicações por ano)}

O Gráfico 1 apresenta a série histórica dos estudos recuperados e selecionados para esta bibliometria. Observa-se que o período de 2008 a 2010 foi importante para as publicações da área de ontologias e tarefas intensivas em conhecimento. $O$ ano de 2008 apresenta 5 publicações, sendo seguido por 2009, com 7 trabalhos recuperados, e 2010 com outros 5 estudos.

Gráfico 1 - Distribuição temporal dos estudos

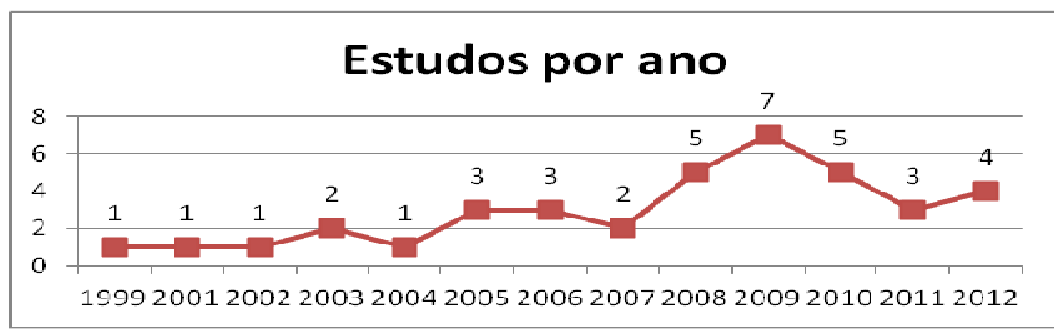

Fonte: Os autores. 
O trabalho pioneiro, identificado pelo levantamento bibliométrico realizado, é intitulado "An Integration Method for the Specification of RuleOriented Mediators", de autoria de Wache, H. et al, e elaborado em 1999. Neste estudo, os autores apresentam uma abordagem baseada em ontologias para a especificação de mediadores. Mediadores são componentes que minimizam a heterogeneidade semântica existente em grandes massas de dados disponíveis na Internet, permitindo o acesso integrado a essas fontes de informações. No escopo deste estudo, os autores afirmam que as ontologias descrevem a semântica do domínio. Por outro lado, afirmam que a especificação dos mediadores e o desenvolvimento de terminologias comuns e compartilhadas são tarefas intensivas em conhecimento e trabalho, e são obstáculos para o uso destes sistemas de mediação. Embora os autores do presente estudo tenham deixado de abordar a tarefa de avaliação enquanto tarefa intensiva em conhecimento, tratam da temática de forma subjacente, dando especial enfoque para as ontologias e sua aplicação para a representação do conhecimento de um determinado domínio.

O estudo mais recente recuperado foi publicado no ano de 2012 por Kumar, denominado "A knowledge Acquisition System for a University". O autor demonstra, no estudo, a estrutura, a facilidade, a interface de usuário e algumas experiências com um sistema de aquisição de conhecimento para armazenamento de processos orientados a conhecimento que são usados por um sistema baseado em conhecimento (Knowledge-Based Systems - KBS) para a área administrativa e educacional. Aborda o conceito de tarefas intensivas em conhecimento demonstrando que elas podem ser objeto de tratamento por este sistema e afirma que as ontologias são empregadas para a representação do conhecimento. Especificamente a tarefa de avaliação, enquanto tarefa intensiva em conhecimento, deixa de ser abordada pelos autores.

\subsection{Principais autores, instituições e países}

Nos 38 estudos analisados, observa-se uma variada lista de autores e instituições: são 106 autores, filiados à 44 instituições de 18 países. A Tabela 4 demonstra os principais autores (com no mínimo 2 publicações) com os seus respectivos números de artigos publicados sobre o tema foco desta investigação, afiliação, cidade e país.

Tabela 4 - Autores com maior número de publicações e sua afiliação

\begin{tabular}{lclll}
\hline \multicolumn{1}{c}{ Autor } & $\begin{array}{c}\text { Número de } \\
\text { publicações }\end{array}$ & \multicolumn{1}{c}{ Afiliação } & Cidade & País \\
\hline Loia, V. & 02 & University of Salerno & Salerno & Itália \\
Yuh-Jen, Chen & 02 & $\begin{array}{l}\text { National Kaohsiung First University of } \\
\text { Science and Technology }\end{array}$ & Kaohsiung & Taiwan \\
Yuh-Min Chen & 02 & National Cheng Kung University & Tainan & Taiwan \\
\hline
\end{tabular}

Fonte: Os autores. 
Observa-se, a partir da análise de coautoria, um relacionamento entre os principais pesquisadores identificados neste estudo, como demonstrado na Figura 2.

Figura 2 - Relações de coautoria
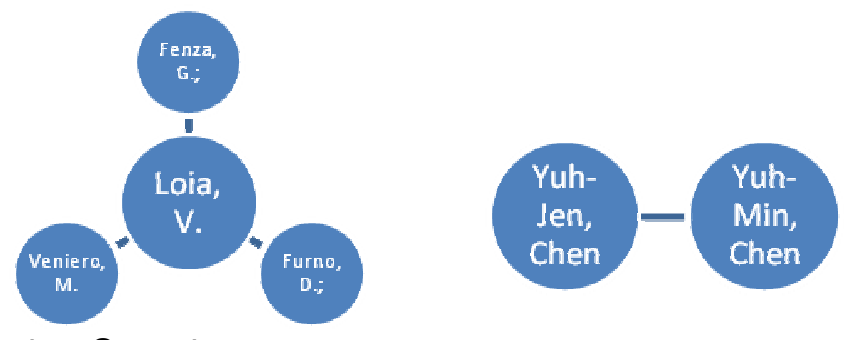

Fonte: Os autores.

Quando as 191 instituições são classificadas por ordem de quantidade de publicações, a National Cheng Kung University, localizada em Taiwan, destaca-se em razão do número de 4 estudos publicados, representando cerca de $10 \%$ dos trabalhos recuperados pela presente pesquisa. Ela é seguida pela Daliann University of technology, da China; Institute of Electrical and Electronics Engineers (IEEE), dos Estados Unidos; e, National Kaohsiung First University of Science and Technology, de Taiwan, todos com duas publicações cada, ou cerca de $5 \%$ dos estudos recuperados cada um (TABELA 5). Essas quatro instituições possuem cerca de $26 \%$ de todas as publicações deste estudo, as demais figuram com apenas uma publicação cada.

Tabela 5 - Instituições com maior número de publicações

\begin{tabular}{|c|c|c|c|}
\hline Instituições & País & Trabalhos & $\%$ \\
\hline National Cheng Kung University & Taiwan & 4 & 10,52 \\
\hline Dalian University of Technology & China & 2 & 5,26 \\
\hline IEEE & Estados Unidos & 2 & 5,26 \\
\hline National Kaohsiung First University of Science and Technology & Taiwan & 2 & 5,26 \\
\hline TOTAL & & 10 & 26,31 \\
\hline TOTAL GERAL & & 38 & $100 \%$ \\
\hline
\end{tabular}

Fonte: Os autores.

\subsection{Principais palavras-chave}

A partir do grupo de trabalhos recuperados, foi possível identificar as 711 palavras-chave verificadas na bibliometria. As palavras-chave "ontologies" (ontologias) e as variações "ontologies (artificial intelligence)" e "ontology" (ontologias - inteligência artificial e ontologia) destacam-se com 65 citações nos estudos analisados. A palavra-chave "humans" (humanos) e "knowledge management" (gestão do conhecimento) são citados com 10 ocorrências cada.

A análise da nuvem de tags demonstrada na Figura 3, gerada a partir das palavras-chave dos trabalhos recuperados, evidencia os termos com maior número de ocorrências

Figura 3 - Nuvem de palavras-chave 


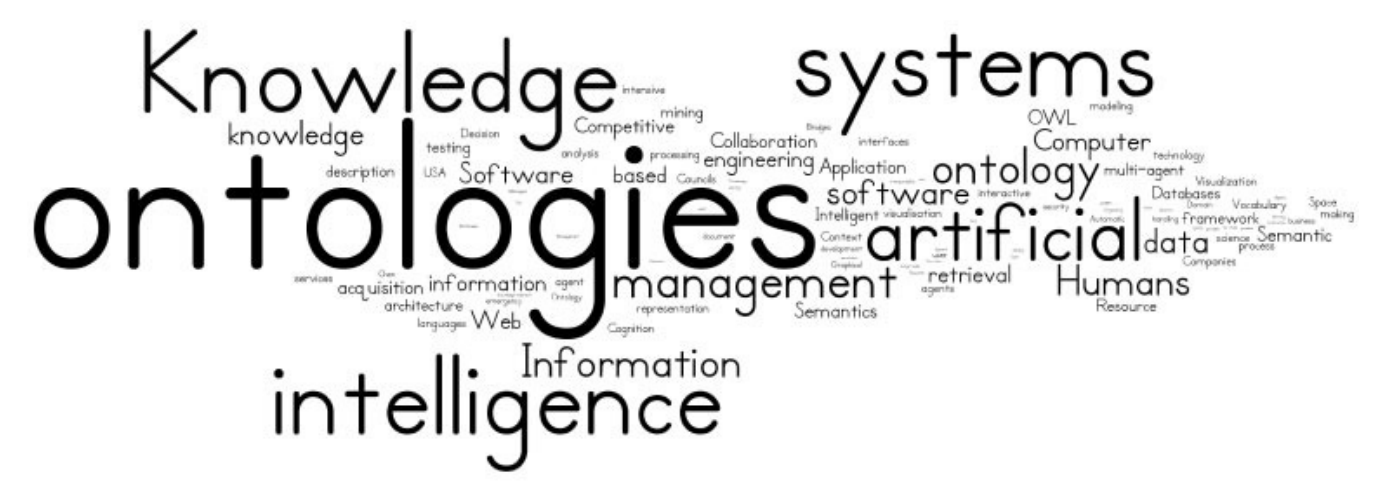

Fonte: Os autores.

Retirando-se os termos "ontologies", "assessment" e "knowledgeintensive task", expressões usadas para a busca dos artigos nas bases indexadas, temos a nuvem de palavras-chave demonstrada na Figura 4, que demonstra quais termos subjacentes são encontrados nos trabalhos recuperados.

Figura 4. Nuvem de palavras-chave

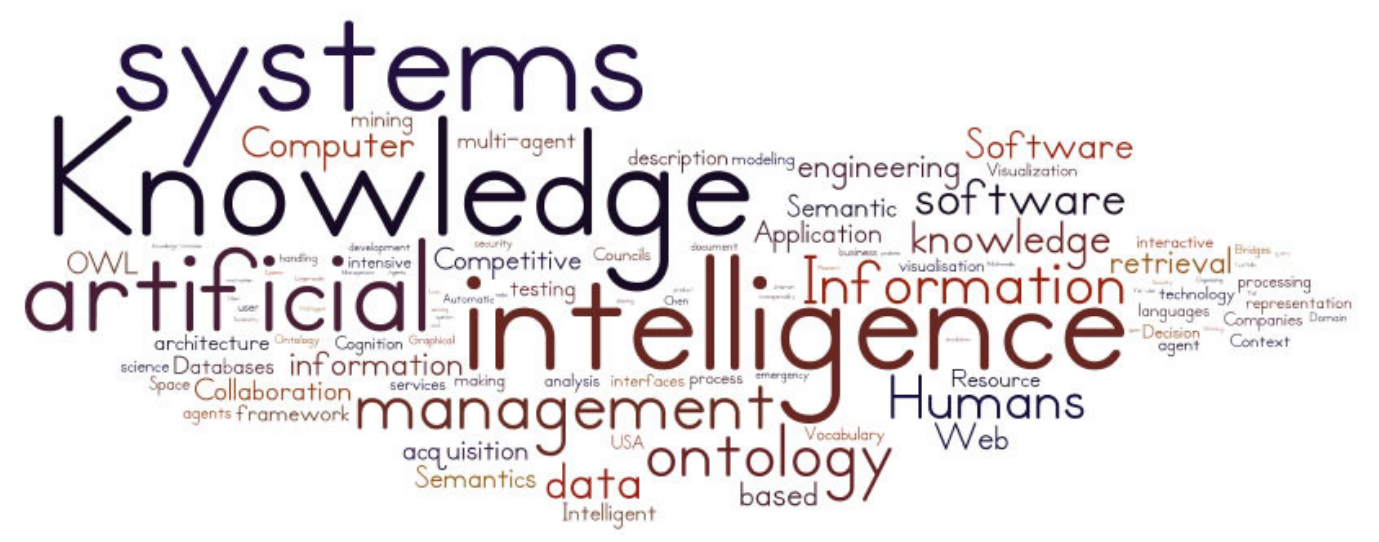

Fonte: Os autores.

\subsection{Trabalhos mais citados}

O trabalho mais citado dentre os estudos recuperados pela presente pesquisa foi escrito por Rubin, D. L. e colaboradores, em 2006, com o título "Using ontologies linked with geometric models to reason about penetrating injuries". O estudo, com 11 citações na base Web of Science e outras 9 citações na base Scopus, apresenta uma metodologia para automatizar o raciocínio sobre ferimentos penetrantes usando ontologias combinadas com dados provenientes de imagens. Os autores tratam a avaliação de ferimentos penetrantes como uma tarefa intensiva em conhecimento, de difícil execução, sendo de vital importância para o atendimento médico do paciente que seja rapidamente executada, para que seja determinado no menor prazo possível o tratamento adequado. As ontologias são empregadas para avaliar os dados geométricos, determinando as consequências do ferimento.

O segundo estudo mais citado tem o título "Ontological foundations for agent support in constructability assessment of steel structures: a case 
study". Este trabalho foi escrito em 2005 por Ugwu e colaboradores, e procura demonstrar a aplicação potencial das ontologias no desenvolvimento de agentes inteligentes baseados em regras para avaliação de construtibilidade em estruturas de aço. Construtibilidade é a condição de um projeto de engenharia ser de fato construído, levando-se em consideração as especificidades do material, do espaço, entre outros. Os autores adotam, no estudo, a definição de ontologias proposta por Gruber (1993), e afirmam que as ontologias são fundamentais para a solução de problemas intensivos em conhecimento e demonstram a tarefa intensiva em conhecimento de avaliação na forma de avaliação de construtibilidade, em um contexto específico de estudo de caso.

\section{Um panorama das publicações científicas pesquisadas}

O objetivo do presente estudo é o de investigar o uso de ontologias como suporte à tarefa intensiva em conhecimento de avaliação, realizando um mapeamento das publicações científicas nesta temática.

O levantamento bibliométrico realizado nas bases científicas indexadas selecionadas, utilizando-se a expressão de busca determinada, resultou na seleção de 38 estudos.

Ao analisarmos estas publicações, oriundas de três importantes bases de dados (Web of Science, Scopus e IEEE), observa-se que em sua grande maioria elas não tratam diretamente da tarefa de avaliação enquanto tarefa intensiva em conhecimento, principalmente quanto o emprego das ontologias para sua implementação.

De maneira geral, o próprio conceito de tarefa intensiva em conhecimento é negligenciado pelos autores nos trabalhos selecionados. Abramovich (2005) trata do estudo da resolução de tarefas (task solving), inclusive com o reconhecimento das ontologias como forma de representação e utilização de experiências humanas, sem abordar as tarefas intensivas em conhecimento. Outros autores tratam da solução de alguns problemas a partir da representação do conhecimento envolvido através das ontologias, mas deixam de abordar claramente as tarefas intensivas em conhecimento (ALEKSY; STEFAN; CUSKE, 2008; AMATO et al, 2010; AMMANN, 2010; AUGELLO et al, 2011; BIENVENIDO; FLORESPARRA, 2003; CHANG et al, 2012; DADZIE; PETRELLI, 2009; DIAZAGUDO; GONZALEZ-CALERO, 2001; DILLON; SIMMONS, 2008; FALKOVYCH, NACK; VAN OSSENBRUGGEN, 2004; FENZ; TJOA; HUDEC, 2009; FENZA et al, 2010; GASEVIC, 2011; GONZALEZ; LOZANO; CASTRO, 2009; HANS et al, 2009; I CHIN; DUEN-REN; WEI-HSIAO, 2005; KOENDERINK; TOP; VAN VLIET, 2006; KOTIS; PAPASALOUROS, 2010; KRIGLSTEIN, 2009; LIU; JIANWEI; XIAOPENG, 2008; LOIA, 2012; MERDAN; VITTORI; KOPPENSTEINER, 2008; PALMA et al, 2002; SHUNHONG; JIAN-HUA, 2006; SMIRNOV; SHILOV; LEVASHOVA, 2007; WACHE et al, 1999; WICKLER; TATE; HANSBERGER, 2007; YUH-JEN; YUH-MIN; YUNG-SHENG, 2009).

Algumas exceções são encontradas nos estudos recuperados. Bienvenido e Flores-Parra (2003) afirmam em seu estudo que o projeto 
configuracional é uma tarefa intensiva em conhecimento. Afirmam que "os melhores designs requerem a aplicação de múltiplas regras com o objetivo de propor, analisar e avaliar as diferentes opções" (BIENVENIDO; FLORES-PARRA, 2003, p. 1). De Boer e Van Vliet (2009), por sua vez, afirmam que a auditoria de produtos de software é uma tarefa intensiva em conhecimento na qual o conhecimento arquitetural ocupa papel principal. Giareth e Ertl (2008) apresentam em seu estudo uma abordagem para o uso de wiki's semânticas com o objetivo de facilitar a busca e a anotação de informações de patentes, considerando, no trabalho, a tarefa de busca e compreensão de patentes como uma tarefa intensiva em conhecimento. Kumar (2012), por sua vez, cita as tarefas intensivas em conhecimento ao demonstrar as tarefas que são controladas pelo sistema baseado em conhecimento desenvolvido e descrito em seu estudo. Rubin et al (2006), ao abordar a avaliação médica de ferimentos penetrantes afirma que esta é uma tarefa extremamente difícil e intensiva em conhecimento, apontando, em seu trabalho, o uso de ontologias e modelos geométricos para o raciocínio sobre ferimentos penetrantes. Ugwu, Anumba e Thorpe (2005), ao propor uma base ontológica para agentes de apoio à avaliação de construtibilidade de estruturas de aço, afirmam que "as ontologias são as fundações nas quais os resolvedores de problemas intensivos em conhecimento se apoiam", reconhecendo que a tarefa objeto de seu estudo é uma tarefa intensiva em conhecimento. Por fim, Yuh-Jen, Yuh-Min e Meng-Sheng (2010) propõem um sistema especialista de recomendação para a consulta de conhecimento empírico sobre produto e afirmam que o ciclo de vida de um produto é um "processo intensivo em conhecimento".

A avaliação, enquanto tarefa intensiva em conhecimento, de forma genérica, é igualmente pouco abordada. De Boer e Van Vliet (2009) tratam em seu estudo da avaliação de qualidade em auditorias de produto de software, propondo uma ontologia que apoia a reutilização dos critérios de qualidade na fase de entrada da auditoria de produtos de software. Neste mesmo sentido é o trabalho de Koenderink, Top e Van Vliet (2006), que se propõe a tarefa intensiva em conhecimento de inspeção através da aplicação de ontologias. Neste estudo, os autores tratam da avaliação de qualidade de verduras como parte do processo de inspeção. Rubin et al (2006), por sua vez, abordam a avaliação no escopo da avaliação médica de ferimentos penetrantes. Ugwu, Anumba e Thorpe (2005) dedicam-se à avaliação de construtibilidade de estruturas de aço, afirmando que muitos do que é projetado não chega a ser executado em razão de limitações do material, razão pela qual os projetos devem ser avaliados sob este ponto de vista.

As ontologias, por sua vez, são amplamente empregadas nos estudos, porém, os conceitos empregados são diversos.

Abramovich (2005), Dillon e Simmons (2008) e Gobin (2011) empregam ontologias partindo da definição do Protégé, ao afirmar que as "ontologias são especificações explícitas dos tipos de recursos que existem e os relacionamentos possíveis entre eles e as instâncias dos conceitos na ontologia" (ABRAMOVICH, 2005, p. 1), ou como empregado por Li, 
Jianwei e Xiaopeng (2008), isto é, "uma ontologia é uma especificação formal e explícita de uma conceitualização compartilhada", definição esta atribuída a Studer et al (1998).

Dadzie e Petrelli (2009), por sua vez, trabalham com o conceito de que as ontologias fornecem definições formais e estruturadas de conceitos e os relacionamentos entre eles, facilitando sua reutilização. Koenderink, Top e Van Vliet (2006) resgatam a ideia de que ontologias são instrumentos próprios para codificar o conhecimento necessário do domínio.

Por fim, o trabalho de Gobin (2011), intitulado "Reusing OWL-S to Model Knowledge Intensive Tasks Performed by Knowledge Based Systems" destaca-se dentre os estudos recuperados na presente pesquisa.

No estudo, o autor desenvolve uma ontologia de tarefa baseada nos modelos de tarefas fornecidos pela metodologia CommonKADS, discute como a modelagem de um sistema baseado em conhecimento é realizado com a metodologia CommonKADS, além de realizar um resgate de OWLS. Além disso, o autor compara construtos de OWL-S e CommonKADS, verificando em que medida ele pode ser utilizado para construir uma ontologia de tarefa para modelar as tarefas que são executadas por um sistema baseado em conhecimento.

Assim, verifica-se, após as análises realizadas, que um número muito pequeno de trabalhos trata da tarefa de avaliação enquanto tarefa intensiva em conhecimento. Desta forma, o presente estudo contribui na medida em que evidencia esta carência na literatura, demonstrando que o uso de ontologias está amplamente consolidado, entretanto, avanços ainda precisam ser feitos na aplicação clara das ontologias no suporte tarefa de avaliação, enquanto tarefa intensiva em conhecimento.

\section{Considerações finais}

Este estudo mapeou e analisou o campo de pesquisas que intersecionam os conceitos de ontologia aplicados ao suporte da tarefa de avaliação enquanto tarefa intensiva em conhecimento. Foi possível delinear um panorama bibliométrico desse campo de estudo, identificando os trabalhos publicados internacionalmente e apontando as tendências dessa literatura.

Depois de superar as dificuldades técnicas para realizar a bibliometria, foi identificado um conjunto de publicações sobre o tema. Foram analisados bibliometricamente as informações de 38 trabalhos, identificando que esses estudos, em geral, não relacionam explicitamente a aplicação de ontologias para o suporte da tarefa de avaliação enquanto tarefa intensiva em conhecimento.

Vê-se, por outro lado, uma ampla utilização das ontologias como forma de representar conhecimento para a solução de diversas tarefas intensivas em conhecimento, entretanto os autores deixam de trata-las de forma explícita. 
Com a análise realizada, o presente trabalho avança na compreensão do relacionamento entre as áreas de ontologias e tarefas intensivas em conhecimento, demonstrando uma carência de estudos que tratem claramente dos conceitos relativos aos modelos de tarefas, bem como da tarefa de avaliação enquanto tarefa intensiva em conhecimento. Ao identificar e listar os principais trabalhos, autores e instituições neste campo de pesquisa, este trabalho também fornece subsídios para a continuidade dos estudos no âmbito internacional, formação de redes de pesquisas interinstitucionais (entre grupos de pesquisas de diferentes instituições, inclusive brasileiras e estrangeiras), desenvolvimento de linhas de pesquisas que estabeleçam e investiguem em profundidade a associação dessas áreas, entre outros aspectos.

A partir dos resultados apresentados, também é possível perceber que o presente trabalho aponta algumas oportunidades para investigações futuras. Alguns trabalhos identificados neste artigo procuram solucionar problemas específicos através da aplicação das ontologias, sem relacionar estes problemas com os modelos de tarefas apontados pela literatura. Desta forma, o desenvolvimento de modelos de solução de tarefas relacionados com o conceito de tarefas intensivas em conhecimento poderia favorecer sua reutilização. Além disto, a abordagem da tarefa de avaliação, neste contexto, poderia permitir o desenvolvimento de novas aplicações com vistas à aprimorar esta atividade a partir do uso das ontologias para a representação do conhecimento.

\section{Referências}

ABRAMOVICH, A. Human experience representation via upper level ontologies. Integration of Knowledge Intensive Multi-Agent Systems. In: INTERNATIONAL CONFERENCE, 2005. [Proceedings...] [S. I.]: [s. n.], 2005. p. 187-190.

ALEKSY, M.; STEFAN, S.; CUSKE, C. A Distributed Simulation Environment for Simulation Modeling in Operational Risk Management. Complex, Intelligent and Software Intensive Systems, Washington, DC: IEEE Computer Soc., p. 126-131, Mar. 2008.

ALMEIDA, E. C. E. O portal de periódicos da Capes: estudo sobre a sua evolução e utilização. 2006. 175 f. Dissertação (Mestrado) - Universidade de Brasília, Brasília. 2006.

ALMEIDA, M. B.; BAX, M. P. Uma visão geral sobre ontologias: pesquisa sobre definições, tipos, aplicações, métodos de avaliação e de construção. Ciência da informação, Brasília, v. 32, n. 3, p. 7-20, 2003.

ALMEIDA, M. B.; MENDONÇA, F. M.; AGANETTE, E. C. Interfaces entre ontologias e conceitos seminais da Ciência da informação: em busca de avanços na organização do conhecimento. In: ENANCIB, v. 4, 2013, Florianópolis. Anais eletrônicos... Disponível em < http://enancib.sites.ufsc.br/index.php/enancib2013/ XIVenancib>. Acesso em: 19 ago. 2014. 
AMATO, F. et al. A Multimedia Document Model for e-Government Information Systems. In: Proceeding CISIS '10 Proceedings of the 2010 International Conference on Complex, Intelligent and Software Intensive Systems. IEEE Computer Society Washington, DC, USA, p. 1099-1104, 2010.

AMMANN, E. A Foundation of Enterprise Knowledge Development. In: INTERNATIONAL CONFERENCE, 5., 2010. Internet and Web Applications and Services (ICIW). [S. I.]: [s. n.] 2010. p. 525-530.

AUGELLO, A. et al. A Modular Framework for Versatile Conversational Agent Building. In: INTERNATIONAL CONFERENCE, 2011. Complex, Intelligent and Software Intensive Systems. [S. I.]: [s. n.], 2011. p. 577582.

BIENVENIDO, J. F.; FLORES-PARRA, I. M. STEM: a methodology for the development of multiagent design tools using a general knowledge model of configurational design. In: INTERNATIONAL CONFERENCE, 2003. Integration of Knowledge Intensive Multi-Agent Systems. [S. I.]: [s. n], 2003. p. 602-607.

CAMPOS, Maria Luiza de Almeida. O papel das definições na pesquisa em ontologia. Perspectivas em Ciência da Informação, Belo Horizonte, v. 15, n. 1, p. 220-238, jan./abr. 2010.

CHANG, L. et al. Reasoning with Large Scale Ontologies in Fuzzy pD* Using MapReduce. Computational Intelligence Magazine, Osaka, Japão, v. 7, n. 2, p. 54-66, 2012.

CHENG-YU, L. et al. Mining Fuzzy Domain Ontology Based on Concept Vector from Wikipedia Category Network. In: IEEE/WIC/ACM INTERNATIONAL CONFERENCE, 2011. Web Intelligence and Intelligent Agent Technology. [S. I.]: [s. n.], 2011. p. 249-252.

DADZIE, A. S.; PETRELLI, D. Visual knowledge exploration and discovery from different points of view. In: IEEE SYMPOSIUM, 2009. Visual Analytics Science and Technology. [S. I.]: [s. n.], 2009. p. 227-228.

DE BOER, R. C.; VAN VLIET, H. QuOnt: an ontology for the reuse of quality criteria. In: SHARK '09 ICSE, 2009. Sharing and Reusing Architectural Knowledge. [S. I.]: [s. n.], 2009. p. 57-64.

DIAZ-AGUDO, B.; GONZALEZ-CALERO, P. A. A declarative similarity framework for knowledge intensive CBR. In: CASE-BASED REASONING RESEARCH AND DEVELOPMENT, 2001. Proceedings... Editors: D. W. Aha and D. Watson, I. [S. I.]: [s. n.], 2001. v. 2080, p. 158-172.

DILLON, T. S.; SIMMONS, G. Semantic Web Support for Open-Source Software Development. In: SITIS '08 IEEE INTERNATIONAL CONFERENCE, 2008. Signal Image Technology and Internet Based Systems. [S. I.]: [s. n.], 2008. p. 606-613.

FALKOVYCH, K. et al. SampLe: towards a framework for systemsupported multimedia authoring. In: INTERNATIONAL MULTIMEDIA 
MODELLING CONFERENCE, 10., 2004. Proceedings... [S. I.]: [s. n.], 2004. p. 362.

FENZ, S.; TJOA, A. M.; HUDEC, M. Ontology-Based Generation of Bayesian Networks. In: CISIS '09 INTERNATIONAL CONFERENCE, 2009. Complex, Intelligent and Software Intensive Systems. [S. I.]: [s. n.], 2009. p. 712717.

FENZA, G. et al. Agent-based Cognitive approach to Airport Security Situation Awareness. In: CISIS 2010 INTERNATIONAL CONFERENCE, 2010. Complex, Intelligent and Software Intensive Systems. [S. I.]: [s. n.], 2010. p. 1057-1062.

GASEVIC, D. An Approach to Folksonomy-Based Ontology Maintenance for Learning Environments. Learning Technologies, IEEE Transactions, Los Alamitos, Califórnia, Estados Unidos, v. 4, n. 4, p. 301-314, 2011.

GIERETH, M.; ERTL, T. Visualization Enhanced Semantic Wikis for Patent Information. In: INTERNATIONAL CONFERENCE,12., 2008. Information Visualisation. [S. I.]: [s. n.], 2008. p. 185-190.

GOBIN, B. A. Reusing OWL-S to model knowledge intensive tasks performed by Knowledge Based Systems. In: INTERNATIONAL CONFERENCE, 9, 2012. ICT \& Knowledge Engineering. [S. I.]: [s. n.], 2012. p. 34-42.

GÓMEZ-PÉREZ, A. Ontological engineering: a state of the art. British Computer Society, Londres, Inglaterra, v. 2, p. 33-43. 1999.

GONZALEZ, J. M.; LOZANO, J. A.; CASTRO, A. Autonomic System Administration. A Testbed on Autonomics. In: ICAS '09 INTERNATIONAL CONFERENCE, 5., 2009. Autonomic and Autonomous Systems. [S. I.]:[s. n.], 2009. p. 117-122.

GRUBER, T. R. A Translation Approach to Portable Ontology Specification. Knowledge Acquisition. Nova York, Estados Unidos, v. 52, n. 6, p. 11111133, 1993.

HAN, L. et al. An Ontology-Based Approach for GUI Testing. In: ANNUAL IEEE INTERNATIONAL COMPUTER SOFTWARE AND APPLICATIONS CONFERENCE, 33., 2009. [Proceedings...]. [S. I.] ; [s. n.], 2009. p. 632633.

KAI-LIANG, C.; JIAU, H. C. Agent-assisted document composition in specific domains. In: INTERNATIONAL CONFERENCE, 2003. Integration of Knowledge Intensive Multi-Agent Systems, 2003. [S. I.]: [s. n.], 2003. p. 444-449.

KOENDERINK, N. J. J. P.; TOP, J. L.; VAN VLIET, L. J. Supporting Knowledge-Intensive Inspection Tasks with Application Ontologies. International Journal of Human Computer Studies, Reino Unido, v. 64, n. 10. p. 974-983. 2006.

KOTIS, K.; PAPASALOUROS, A. Learning Useful Kick-off Ontologies from Query Logs: HCOME Revised. In: INTERNATIONAL CONFERENCE, 2010. 
Complex, Intelligent and Software Intensive Systems. [S. I.]: [s. n.], 2010. p. 345-351.

KRIGLSTEIN, S. User Requirements Analysis on Ontology Visualization. In: CISIS '09 INTERNATIONAL CONFERENCE, 2009. Complex, Intelligent and Software Intensive Systems. [S. I.]: [s. n.], 2009. p. 694 - 699.

KUMAR, S. A Knowledge Acquisition System for a university educational process. In: IEEE INTERNATIONAL CONFERENCE, 7., 2012. Industrial and Information Systems. [S. I.]: [s. n.], 2012. p. 1-6.

LI, Q.; JIANWEI, W.; XIAOPENG, W. Construction Method of Knowledge Base Based on Fuzzy and Modular Ontology. In: PACIIA '08 PACIFIC-ASIA WORKSHOP, 2008. Computational Intelligence and Industrial Application, 2008. [S. I.]: [s. n.], 2008. p. 70-74.

LOIA, V. CISIS 2012 Keynote. In: INTERNATIONAL CONFERENCE, 6., 2012. Complex, Intelligent and Software Intensive Systems. [S. I.]: [s. n.], 2012. p. xxxvii-xxxvii.

MERDAN, M. et al. Simulation of an ontology-based multi-agent transport system. In: SICE ANNUAL CONFERENCE, 2008. [Proceedings...]. [S. I.]: [s. n. ], 2008. p. 3339-3343.

PALMA, J. et al. ACUDES: architecture for intensive care units decision support. In: ANNUAL CONFERENCE, 24.; ANNUAL FALL MEETING OF THE BIOMEDICAL ENGINEERING SOCIETY EMBS/BMES CONFERENCE, 2., 2002. Engineering in Medicine and Biology. [S. I.]: [s. n.], 2002. v. 3, p.1938-1939.

RAUTENBERG, S.; GAUTHIER, F. O.; LOTTIN, P.; DUARTE, C. E. J.; TODESCO, J. L. ontoKEM: uma ferramenta para construção e documentação de ontologias. In: SEMINÁRIO DE PESQUISA EM ONTOLOGIA NO BRASIL. 1., 2008, Niterói. Anais... Niterói: Universidade Federal Fluminense, 2008.

RAUTENBERG, Sandro et al. Ferramenta ontoKEM: uma contribuição à Ciência da Informação para o desenvolvimento de ontologias. Perspectivas em Ciência da Informação, Belo Horizonte, v. 15, n. 1, p. 239-258, jan./abr. 2010.

RUBIN, D. L. et al. Using ontologies linked with geometric models to reason about penetrating injuries. Artificial Intelligence in Medicine, Viena, Áustria, v. 37, n. 3, p. 167-176, 2006.

SANTOS, R. N. M.; KOBASHI, N. Y. Bibliometria, cientometria, infometria: conceitos e aplicações. Pesq. Bras. Ci. Inf., Brasília, v.2, n.1, p. 155-172. 2009.

SCHREIBER, G. et al. Knowledge Engineering and Management: the CommonKADS Methodology. Cambridge. Mass,: MIT Press, 2002.

SHUN-HONG, S.; JIAN-HUA, Y. Automatic Ontology Generation Using Schema Information. In: IEEE/WIC/ACM INTERNATIONAL CONFERENCE, 2006. Web Intelligence, [S. I.]: [s. n.], 2006. p. 526-531. 
SMIRNOV, A.; SHILOV, N.; LEVASHOVA, T. Cooperative Self-Organizing Coalitions: Technological Framework. In: INTERNATIONAL CONFERENCE, 2007. Integration of Knowledge Intensive Multi-Agent Systems. [S. I.]: [s. n.], 2007. p. 396-400.

STUDER, R. Situation and Perspective of Knowledge Engineering. In: SCIENTIFIC Literature Digital Library. [S. l.]: [s. n.], 2000.

STUDER, R.; BENJAMINS, R.; FENSEL, D. Knowledge Engineering: Principles and Methods. Data \& Knowledge Engineering, Los Angeles, Estados Unidos, v. 25, p. 161-197, 1998.

UGWU, O. O.; ANUMBA, C. J.; THORPE, A. Ontological foundations for agent support in constructability assessment of steel structures - A case study. Automation in Construction, College Park, Estados Unidos, v. 14, n. 1 , p. 99-114, 2005.

WACHE, $H$. et al. An integration method for the specification of ruleoriented mediators. In: DANTE '99 1999 INTERNATIONAL SYMPOSIUM. 1999. Database Applications in Non-Traditional Environments. [S. I.]: [s. n.], 1999. p. 109-112.

WICKLER, G.; TATE, A.; HANSBERGER, J. Supporting Collaborative Operations within a Coalition Personnel Recovery Center. In: KIMAS 2007 INTERNATIONAL CONFERENCE, 2007. Integration of Knowledge Intensive Multi-Agent Systems, [S. I.]: [s. n.], 2007. p. 378-383.

WU, I. C.; DUEN-REN, L.; WEI-HSIAO, C. Task-stage knowledge support: coupling user information needs with stage identification. Information Reuse and Integration, Conf, 2005. IRI -2005 IEEE International Conference on., 2005. 15-17 Aug. 2005. p.19-24. 2005.

YUH-JEN, C.; YUH-MIN, C.; MENG-SHENG, W. An expert recommendation system for product empirical knowledge consultation. Computer Science and Information Technology (ICCSIT), 2010 3rd IEEE International Conference on, 2010. 9-11 July 2010. p.23-27. 2010.

YUH-JEN, C.; YUH-MIN, C.; YUNG-SHENG, S. An Ontology-Based Distributed Case-Based Reasoning for Virtual Enterprises. Complex, Intelligent and Software Intensive Systems, 2009. CISIS '09. International Conference on, 2009. 16-19 March 2009. p.128-135. 2009. 